\title{
Distribution et extension de la résistance chloroplastique aux triazines chez les adventices annuelles en France
}

\author{
Jacques GASQUEZ $(*)$, Gilbert BARRALIS $\left({ }^{*}\right)$ \& Nicole AIGLE $\left({ }^{* *}\right)$ \\ (*) I.N.R.A., Laboratoire de Malherbologie, BV 1540, F 21034 Dijon Cedex. \\ (**) A.G.P.M., 1, Place S. de Lestapis, F 64000 Pau.
}

RÉSUMÉ

Mauvaise herbe,

Résistance aux triazines,

Résistance chloroplastique.
La résistance chloroplastique aux triazines a été observée en France chez 13 espèces, dont les auteurs précisent l'importance agronomique et la distribution géographique ; les 3 espèces qui présentent la plus grande dispersion sont Amaranthus retroflexus L., Chenopodium album L. et Solanum nigrum L.

\section{SUMMARY}

Weeds,

Triazine resistance,

Chloroplastic resistance.

\section{Distribution and spread of chloroplast-based triazine resistance in annual weeds in France}

Triazine-resistant populations have been reported within 13 species in France. Their agronomic importance and geographical distribution is given. The 3 most widen distributed species are Amaranthus retroflexus L., Chenopodium album $\mathrm{L}$. and Solanum nigrum $\mathrm{L}$.

\section{INTRODUCTION}

Si pendant plusieurs années la culture du maïs a été largement facilitée par l'utilisation d'herbicides très efficaces (atrazine notamment), récemment, en différentes régions d'Europe et d'Amérique, se sont développées des populations de mauvaises herbes insensibles aux triazines.

La résistance à l'atrazine de certaines graminées cultivées et de quelques graminées adventices (généralement de la sous-famille des Panicoïdées) est connue depuis longtemps comme la conséquence d'une plus ou moins grande capacité de métabolisation de l'herbicide. Ces espèces possèdent une ou plusicurs voies de détoxication alors que la majorité des mauvaises herbes, surtout dicotylédones, en sont dépourvues. En 1977, SoUZA-MACHADO et al. montrent, chez Chenopodium album L., l'existence d'individus dont les chloroplastes sont insensibles aux triazines. Ces plantes, appartenant à une espèce normalement sensible, résistent à des doses d'herbicide extrêmement élevées. Depuis, de telles populations ont été recensées chez diverses espèces dans de nombreuses régions (GRESSEL et al., 1981).

En 1979, nous avions réalisé une enquête pour déterminer l'importance du phénomène (AIGLE, BARRALIS \& GasqueZ, 1980). Depuis, la résistance chloroplastique a été décrite chez de nouvelles espèces et les espèces antérieurement décrites ont gagné de nouvelles régions et de nouvelles cultures.

Nous actualisons dans cette mise au point, notre connaissance de l'extension du phénomène. Son importance nous a amenés à étendre notre prospection hors des cultures de maïs, à des zones partiellement ou régulièrement désherbées aux triazines, comme le vignoble.

\section{MATÉRIEL ET MÉTHODES}

Nous avons utilisé un matériel indifféremment constitué de semences ou de plantules récoltées dans les stations d'origine et même d'échantillons de sols mis en serre dans de bonnes conditions de germination pour obtenir une levée satisfaisante des espèces à éprouver. Les tests ont été réalisés soit par le traitement de plantules à forte dose d'atrazine $(2000 \mathrm{~g}$ m.a./ha), soit par l'observation de la fluorescence de feuilles isolées, selon un protocole déjà décrit, ou encore par la mesure de l'activité de chloroplastes isolés (DUCRUET \& GASQUEZ, 1978).

\section{RÉSULTATS}

\section{A. Espèces nouvellement résistantes}

Tant en Amérique (USA et Canada) qu'en Europe, la résistance avait déjà été décrite dans 9 pays, chez 16 espèces ; il est probable que ce phénomène puisse exister dans d'autres pays d'Europe. A l'exception de Bromus tectorum L. cité par GRESSEL (GRESSEL et al., 1981), il ne semble pas que la résistance ait été découverte chez de nouvelles espèces hors de France depuis 1979. En revanche, nous 
TABLEAU 1

Distribution géographique des espèces chloroplastiquement résistantes

(Les localisations des nouvelles espèces sont référencées).

Geographical distribution of triazine-resistant species

(Locations of the new French species are listed).

\begin{tabular}{|c|c|c|c|c|c|c|c|c|}
\hline & $\begin{array}{l}\text { U.S.A. + } \\
\text { Canada }\end{array}$ & Allemagne & Autriche & $\begin{array}{l}\text { Grande- } \\
\text { Bretagne }\end{array}$ & Italie & Suisse & Hongrie & France \\
\hline $\begin{array}{l}\text { Amaranthus spp.* } \\
\text { Amaranthus graezicans L. } \\
\text { Amaranthus lividus L. } \\
\text { Ambrosia artemisiifolia L. } \\
\text { Bidens tripartita L. } \\
\text { Brassica campestris L. } \\
\text { Bromus tectorum L. } \\
\text { Chenopodium album L. } \\
\text { Chenopodium polyspermum L. } \\
\text { Chenopodium strictum Roth. } \\
\text { Echinochloa crus g. (L.) P.B. } \\
\text { Poa annua L. } \\
\text { Polygonum lapathifolium L. } \\
\text { Polygonum persicaria L. } \\
\text { Senecio vulgaris L. } \\
\text { Setaria faberi Hermann } \\
\text { Setaria pumila (Poir.) Rets. } \\
\text { Setaria viridis (L.) P.B. } \\
\text { Solanum nigrum L. } \\
\text { Sonchus asper (L.) Hill } \\
\text { Stellaria media (L.) Vill. }\end{array}$ & $\begin{array}{l}+ \\
+ \\
+ \\
+ \\
+\end{array}$ & $\begin{array}{l}+ \\
+\end{array}$ & + & + & + & $\begin{array}{l}+ \\
+ \\
+\end{array}$ & + & $\begin{array}{l}+ \\
+ \\
+\left({ }^{2}\right) \\
+ \\
+ \\
+ \\
+\left({ }^{3}\right) \\
+\left({ }^{4}\right) \\
+\left(^{(5)}\right. \\
+ \\
+ \\
+\left({ }^{6}\right)\end{array}$ \\
\hline
\end{tabular}

* Confondues: A. retroflexus L. ; A. bouchonii Thell. ; A. hybridus L. ; A. cruentus L. ; A. powellii S. Wats

(1) A. bouchonii - 63-Neschers - Maïs.

(2) 64 - Miossens - Maïs.

(3) 51 - St-Martin-d'Allois - Vigne.

( $\left.{ }^{4}\right) 16$ - Aigre - Mais.

(s) 16 - St-Fraigne - Maïs.

(6) 30 - St-Cristol-les-Ales - Vigne.

avons d'abord observé dans une vigne de Champagne une population de Senecio vulgaris L. et dans une vigne du Gard, une population de Sonchus asper (L.) Hill. résistant au champ à de très fortes doses de triazines et dont les individus présentent des courbes de fluorescence identiques à celles que nous avons rapportées pour les autres plantes résistantes (DUCRUET \& GASQUEZ, 1978; GASOUEZ \& BARRALIS, 1979 ; GASQUEZ \& COMPOINT, 1980).

Toutes les espèces déjà connues, surtout dicotylédones, sont des plantes qui ne détoxifient pas ou très peu; cependant nous avons découvert la résistance chloroplastique chez des graminées présentant une certaine détoxication. Nous l'avons vérifiée, par fluorescence, pour la seule population actuellement découverte pour chaque espèce (tableau 1, fig. 4) chez Setaria faberi Hermann, Setaria pumila (Poiret) R. et S., Echinochloa crus galli (L.) P. B. et, par mesure de l'activité de chloroplastes isolés, chez Setaria viridis (L.) P. B. (GAsQuez \& COMPOINT, 1981a). Il existe peut-être d'autres populations de ce type chez les Panicoïdées, mais le niveau de résistance dû à la détoxication rend la manifestation de la résistance chloroplastique moins évidente au champ.

Dans le tableau 1, nous avons fait figurer les espèces dont nous avons directement vérifié la résistance ou dont l'auteur a donné les caractéristiques. En effet, la résistance chez d'autres espèces a été rapportée sans avoir été vérifiée par un test approprié ; c'est le cas d'Atriplex patula L. et de Chenopodium ficifolium $\mathrm{Sm}$. en Allemagne, d'Erigeron canadense L. en Suisse ; ces populations résisteraient à de très fortes doses d'atrazine. D'autre part, en Grande-Breta- gne, il semble que la résistance chez Senecio squallidus L. (GRESSEL et al., 1981) n'ait jamais existé (ScOTT, comm. pers.).

Les amaranthes dressées à fleurs pentamères posent un problème d'ordre systématique, car il existe de nombreuses formes morphologiques intermédiaires entre les types décrits. Ainsi, les auteurs américains ont d'abord cité comme résistante une population d'Amaranthus retroflexus L. (BANDEEN et al., 1979) qui s'est avérée par la suite appartenir à A. hybridus L. (AHRENS et al., 1981). Au Canada, la résistance a été rapportée chez $A$. retroflexus $\mathrm{L}$. et $A$. powellii S. Wats (WARWICK \& WEAVER, 1980), en Italie chez un biotype proche d'A. cruentus L.. En France, nous avons d'abord observé la résistance de biotypes rapportés à $A$. retroflexus s.l. (GASQUEZ \& COMPOINT, 1980), puis à $A$. hybridus (AIGLE et al., 1980) et maintenant à $A$. bouchonii Thell. C'est pourquoi, en raison de l'extrême proximité génétique que nous avons déjà signalée pour ces espèces (BARRALIS et al., 1978) et à cause de leur comportement écologique très voisin, nous préférons considérer ces amaranthes comme faisant toutes partie d'un même ensemble.

\section{B. Extension des espèces résistantes déjà connues}

Pour des raisons certainement d'ordre génétique, certaines espèces comme Chenopodium album L., Amaranthus spp. et Solanum nigrum L. présentent, dans tous les pays où on les rencontre, de nombreuses populations résistantes largement distribuées. Ainsi en Hongrie les zones touchées 
Figure 1

Répartition des populations résistantes de Solanum nigrum $L$. en fonction des surfaces cultivées en maïs, par département.

Distribution of resistant populations of Solanum nigrum L. in relation to area under maize.

Superficie en maïs par département Area under maize in each department

$\square$ de 0 à 10000 ha

$\nabla$ de 10000 à 30000 ha

Ua de 30000 à 50000 ha

de 50000 à 70000 ha

plus de 70000 ha

\section{Importance des surfaces infestées \\ Infested area}

- Jusqu'à 1000 ha

- de 1000 à 5000 ha

$\triangle$ de 5000 à 10000 ha

$\triangle$ de 10000 à 20000 ha

plus de 20000 ha

- Population de vignoble

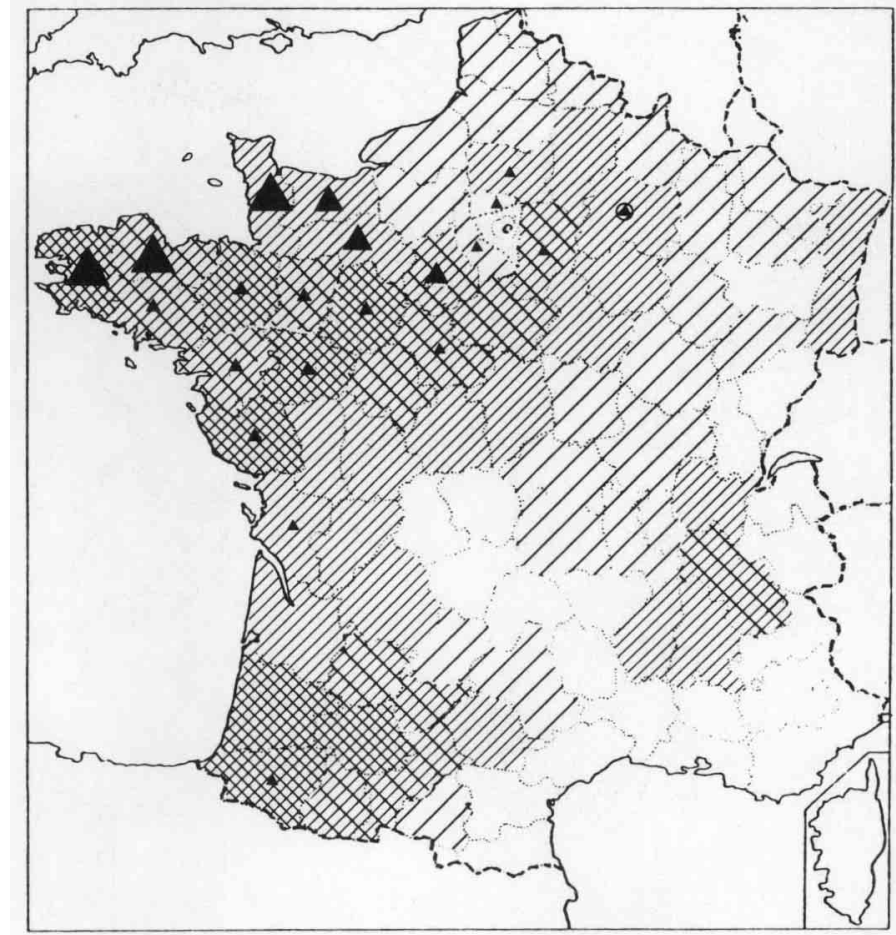

Figure 2

Répartition des populations résistantes de Chenopodium album $L$. en fonction des surfaces cultivées en maïs, par département.

Distribution of resistant populations of Chenopodium album $L$. in relation to area under maize.

(Légende cf. figure 1) - (Caption see map 1) représenteraient 80 p. 100 des surfaces en maïs (GRESSEL et al., 1981). En revanche, pour les autres espèces, les biotypes résistants restent localisés, ne s'étendant qu'accidentellement.

En France, ces 3 principales espèces infestent actuellement environ 200000 ha.

Solanum nigrum L. est l'espèce aujourd'hui la plus largement répandue dans les maïs (environ 125000 ha dans 12 départements). Sa présence est signalée aussi dans 9 autres départements (fig. 1). La distribution surtout atlantique des zones infestées est directement liée à l'abondance de l'espèce dans ces régions. L'extension de la résistance chez cette espèce est certainement due à sa grande productivité de semences et à l'importance du transport accidentel de graines (par les baies collées aux instruments, par les lisiers ou les fumiers, par les oiseaux). Cependant toutes les populations résistantes ne sont pas identiques; en Bretagne il existe par exemple, des biotypes de Solanum nigrum à baies noires et d'autres à baies jaunes.

Chenopodium album L. est l'espèce dont la distribution géographique est la plus grande (26 départements touchés) ; cependant la quasi-totalité des surfaces (environ 53000 ha) se situe dans 9 départements seulement (fig. 2). Bien que les populations résistantes existent dans des régions plus diversifiées, la moindre importance spatiale de ces populations est peut-être due à la faible dispersion des semences. A cette diversité géographique, peut être corrélée une diversité d'origine génétique (GASQUEZ \& COMPOINT, 1981b).

L'ensemble des Amaranthus spp. représente environ 


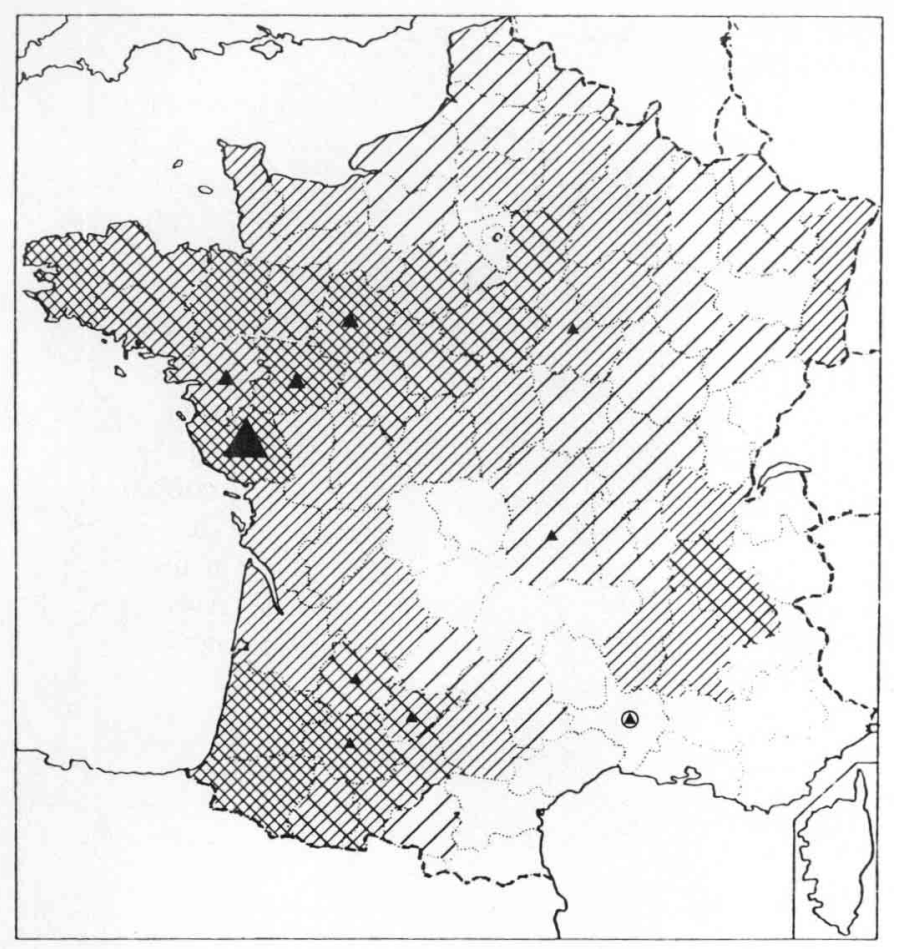

Figure 3

Répartition des populations résistantes d'Amaranthus spp. en fonction des surfaces cultivées en maïs, par département.

Distribution of resistant populations of Amaranthus spp. in relation to area under maize.

\section{(Légende cf. figure 1) - (Caption see map 1)}

\section{Figure 4}

Localisation des populations résistantes d'espèces peu représentées. Location of resistant populations of minor importance.

$\square$ Chenopodium polyspermum L.

$\triangle$ Echinochloa crus galli (L.) P.B.

O Poa annua L.

$\diamond$ Polygonum lapathifolium L.

- Polygonum persicaria L.
Senecio vulgaris $\mathrm{L}$.

$\nabla$ Setaria faberi Hermann

$\checkmark$ Setaria pumila (Poiret)

R. et $S$.

- Setaria viridis (L.) P.B.

- Sonchus asper (L.) Hill

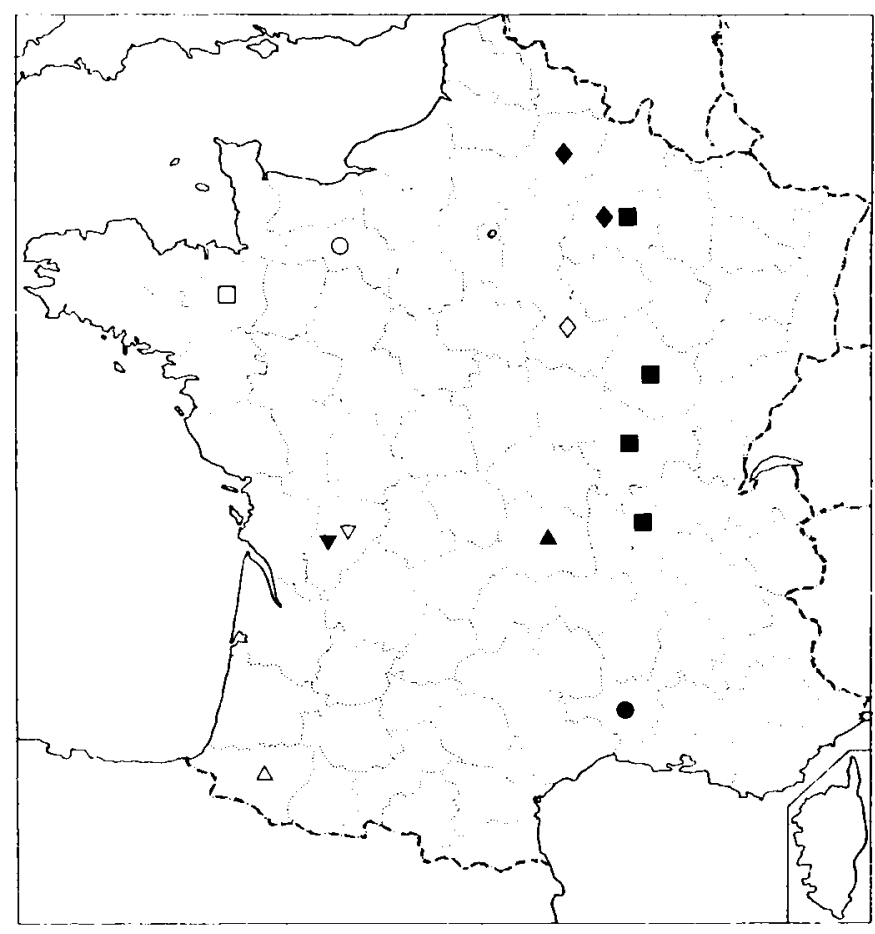

25000 ha avec une nette prédominance des populations de type $A$. retroflexus s.l. (fig. 3 ). La moindre extension de cette adventice ( 8 départements seulement) et sa distribution plus méridionale tiennent certainement à ses caractéristiques écologiques liées à ses exigences thermiques pour la germination.

Des populations résistantes de ces 3 mauvaises herbes existent également dans des vignes de Champagne (fig. 1) et du Gard (fig. 2 et 3). Malgré la variété des produits utilisables dans cette culture, il semble que la sélection soit toujours due à la simazine bien que les plantes résistent aussi à d'autres triazines cmployées dans le vignoble.

10 autres espèces, dont les populations résistantes semblent limitées (fig. 4), sont inégalement réparties dans les zones urbanisées (Poa annua L.), dans les vignes (Senecio vulgaris L., Sonchus asper (L.) Hill.) et dans les maïs pour les autres.

Ainsi, Polygonum lapathifolium L. infeste uniquement un village de l'Yonne (environ 1000 ha) et s'étend lentement par les bords de chemins ; Polygonum persicaria L. occupe une surface un peu supérieure ( 2 à 3000 ha) dans les maïs de l'Aisne. La présence de $P$. persicaria $L$. dans les vignes de Champagne, à proximité du foyer de l'Aisne, pourrait être due à l'extension accidentelle de celui-ci.

Chenopodium polyspermum L. a été rencontré dans quelques rares stations autour de Rennes et Poa annua L. dans une seule localité de l'Orne. Il semble toutefois que cette dernière espèce ait posé (ou pose encore) des problèmes dans différentes villes de l'Ouest et du Jura; mais comme le problème ne se pose que dans des zones non 
cultivées, l'utilisation d'associations d'herbicides puissants, à forte dose, permet l'élimination des résistants éventuels. Enfin Senecio vulgaris semble actuellement en forte extension dans le vignoble du Beaujolais et de Bourgogne.

\section{CONCLUSION}

Sur le territoire français, le phénomène de résistance chloroplastique est maintenant connu chez 13 espèces dont 8 n'ont jamais été signalées ailleurs. Ceci peut être dû à la grande diversité pédoclimatique des milieux cultivés ou rudéraux désherbés aux triazines. Pour chacune d'elles on connaît un certain nombre de populations résistantes dont l'importance et la distribution géographique ne semblent pas corrélées à la répartition et à l'abondance de l'espèce en France.

Cette évolution s'est essentiellement produite dans les champs de maïs qu'ils soient cultivés pour le grain ou pour le fourrage, mais l'extension du désherbage chimique du vignoble a déjà contribué à la sélection de telles populations.

Les plus grandes surfaces touchées se situent dans les régions de maïziculture, mais bien que des cas de résistance aient été signalés dans le Sud-Ouest, c'est dans l'Ouest que le phénomène est le plus étendu : la Bretagne, la Normandie, les Pays de Loire et l'Ouest du Bassin Parisien. Les 3 principales espèces infestent dans ces régions 200000 ha sur les 800000 environ cultivés en maïs.

Le phénomène de résistance chloroplastique semble très général puisque de très nombreuses familles de mauvaises herbes sont déjà touchées. Il n'est pas possible de dire que l'inventaire est clos, tant pour l'extension géographique des espèces citées que pour le nombre des espèces résistantes.

Cette résistance particulière peut être considérée comme la manifestation d'une évolution brutale et extrême sous l'effet d'un facteur prépondérant du milieu, agissant de manière stable et répétée. Sans que l'on puisse préciser les modalités de l'apparition du phénomène, il est cependant directement lié à la monoculture ou à une rotation très simplifiée toujours désherbée avec le même produit persistant. L'absence d'individus résistants dans certaines régions de maïziculture n'est peut-être que temporaire. La résistance pourrait avoir pour origine soit une contamination accidentelle à partir de régions voisines, soit le développement d'éventuels résistants indigènes qui n'auraient pas encore rencontré les conditions de milieu favorables.

La très grande valeur adaptative des individus résistants et l'absence de concurrence dans les cultures désherbées leur a permis de se développer d'une manière extraordinaire, les transformant en véritable fléau.

Les risques d'extension au champ des populations déjà résistantes semblent en étroite relation avec leur aptitude à produire un nombre élevé de semences facilement disséminées, puisque le caractère est transmis par le cytoplasme maternel.

La présence de ces populations résistantes est toujours révélée par la répétition d'un traitement chimique persistant ; pour en éviter l'extension, il faut choisir un système de culture qui permette l'usage de traitements chimiques efficaces contre ces mauvaises herbes.

Reçu le 8 juillet 1981. Accepté le 15 octobre 1981.

\section{RÉFÉRENCES BIBLIOGRAPHIQUES}

\begin{abstract}
Ahrens W. H., Wax L. M., Stoller E. W., 1981. Identification of triazine - resistant Amaranthus spp. Weed Sci. 29 (3), 345-348.

Aigle N., Barralis G., Gasquez J., 1980. Quelques données sur la répartition de la résistance chloroplastique en France. $V I^{*}$ Coll. Intern. Ecologie, Biologie, Systématique des Mauvaises Herbes, 367376.
\end{abstract}

Bandeen J. D., Garochetti J. V., Ryan G. F., Maltais B., 1979. Discovery and distribution of triazine resistant weeds in North America. Abstr. Weed Sci. Soc. Am., 108.

Barralis G., Chadœuf R., Compoint J. P., Gasquez J., Lonchamp J. P., 1978. Etude de quelques aspects de la dynamique d'une agrophytocénose. Symposium Medit. de Herbicidas, 85-98.

Ducruet J. M., Gasquez J., 1978. Observation de la fluorescence sur feuille entière et mise en évidence de la résistance chloroplastique à l'atrazine chez Chenopodium album et Poa annua. Chemosphère 8, 691-696.

Gasquez J., Barralis G., 1979. Mise en évidence de la résistance aux triazines chez Solanum nigrum L. et Polygonum lapathifolium L. par observation de la fluorescence de feuilles isolées. C.R. Acad. Sci. Paris, Séric D, 1391-1393.

Gasquez J., Compoint J. P., 1980. Trois nouvelles mauvaises herbes résistant aux triazines en France: Amaranthus retroflexus s.l., Chenopodium polyspermum L., Polygonum persicaria. Chemosphère 9, 39-43.

Gasquez J., Compoint J. P., 1981a. Observation de chloroplastes résistants aux triazines chez une panicoïdće Setaria viridis L. Agronomie 1, (10) 923-926.

Gasquez J., Compoint J. P., 1981b. Isoenzymatic variations in populations of Chenopodium album L. resistant and susceptible to triazines. Agroecosystem 7, 1-10.

Gressel J., Ammon H. U., Fogelfors H., Gasquez J., Kay Q. O. W., Kees H., 1981. Discovery and distribution of herbicide resistant weeds outside North America in Herbicide Resistance in Plants Ed. by Lebaron H., Gressel J., Wiley Inc. N.Y. (in press).

Souza-Machado V., Bandeen J. D., Stephenson G. R., Jensen K. I. N., 1977. Differential atrazine interference with the Hill reaction of isolated chloroplasts from Chenopodium album biotypes. Weed Res. 17, 407-413.

Warwick S. I., Weaver S. E., 1980. Atrazine resistance in Amaranthus retroflexus (redroot pigweed) and $A$. powellii (green pigweed) from southern Ontario. Can. J. Pl. Sci. 60, 1485-1488. 